\title{
Possible Effects of Chronic Otitis Media with and without Cholesteatoma on Bone Conduction Thresholds: An Evaluation of 112 Cases
}

\author{
Cagatay Han Ulku', Demet Aydogdu², Abitter Yucel ${ }^{1}$, Fuat Aydemir ${ }^{1}$ \\ 1 Department of Otolaryngology Head and Neck Surgery, Necmettin Erbakan University Meram Medical Faculty, Konya, Turkey \\ 2 Department of Radiology, Necmettin Erbakan University Meram Medical Faculty, Konya, Turkey \\ Cagatay Han Ulku, ORCID: 0000-0001-6230-9212 \\ Demet Aydogdu, ORCID: 0000-0001-7643-5084 \\ Abitter Yucel, ORCID: 0000-0002-6433-0362 \\ Fuat Aydemir, ORCID: 0000-0001-6699-0420
}

\begin{abstract}
Objective: The aim of this study is to evaluate any possible effects of chronic otitis media (COM), with or without accompanying cholesteatoma, on bone conduction thresholds (BCT).

Methods: A total of 112 cases who underwent surgery for unilateral COM between 2006 and 2014 were enrolled in the study. Eighty cases had no cholesteatoma (Group 1). Thirty-two cases did have cholesteatoma (Group 2). Prior to surgery, the temporal bone was evaluated radiologically by use of high resolution computed tomography. The presence of a clinically and radiologically normal contralateral ear was the principal selection criterion for the cases. BCT at $0.5,1,2,4 \mathrm{kHz}$ and their averages were evaluated with pure tone audiogram (PTA) and the normal and diseased ears in each group were compared. This comparison was also made between the diseased ears in Groups 1 and 2.
\end{abstract}

Results: There was a statistically significant difference observed between the mean BCT scores obtained by PTA for the normal/diseased ears in Group $1(9.78 \pm 0.98 / 17.34 \pm 1.71$ $\mathrm{dB})$ and in Group $2(9.10 \pm 0.99 / 17.58 \pm 2.59 \mathrm{~dB})$. This statistically significant difference was observed for each of the four different frequencies $(p<0.0001)$. However, there was no statistically significant difference observed between the mean BCT scores obtained by PTA for the diseased ears in Groups 1 and $2(17.34 \pm 1.71 / 17.58 \pm 2.59 \mathrm{~dB})$. Similarly, in the comparison between the diseased ears, the differences of BCT at all four different frequencies $(0.5,1,2,4 \mathrm{kHz})$ lacked statistical significance ( $p>0.05)$.

Conclusion: As a result of this study, we can say that COM may lead to sensorineural hearing loss. However, we observed that the presence of cholesteatoma does not exert an additional negative effect on cochlear function.

Keywords: Chronic otitis media, cholesteatoma, sensorineural hearing loss. 


\section{Introduction}

Chronic otitis media (COM) is a highly prevalent disease worldwide. Its prevalence in developing countries is as high as 72 cases per 1000 inhabitants. Although hearing loss is the main health issue in COM, the complications of the disease may lead to death. ${ }^{[1]}$

Whilst COM is usually associated with conductive hearing loss, it may also cause sensorineural hearing loss (SNHL). ${ }^{[2-5]}$ Conductive hearing loss due to COM is extensively discussed in the literature, but the relationship between SNHL and COM remains controversial. ${ }^{[1]}$ Inflammatory mediators and lytic enzymes produced by the reactive middle ear epithelium are thought to be the main reason for SNHL in cases of COM. These molecules pass through the round window membrane and cause destruction of hair cells. ${ }^{[6-8]} \mathrm{COM}$ with accompanying cholesteatoma is a more destructive process than COM on its own. Thus, it is hypothesized that cochlear damage might be more evident in cases involving cholesteatoma. ${ }^{[9,10]}$

The aim of this study, then, is to evaluate the relationship between COM and the bone-conduction threshold. We also investigate whether the presence of cholesteatoma may have an additional negative effect on cochlear function.

\section{Material and Methods}

The files of patients undergoing surgery between 2006 and 2014 for either unilateral COM without cholesteatoma (Group 1) (n=80), or unilateral COM with cholesteatoma (Group 2) (n=32), were retrospectively reviewed. Ethical approval was first obtained from the hospital ethics committee (Necmettin Erbakan University Meram Medical Faculty, Ethics Committee Decision - 017/777). In all cases, the temporal bone had been evaluated radiologically by high resolution computed tomography prior to surgery. The key selection criterion for inclusion in the study was that patients should have a clinically and radiologically normal contralateral ear (Figure 1). Previous ear surgery, a history of severe head or acoustic trauma, the presence of an otic capsule erosion revealed either radiologically or intraoperatively, or perilymphatic fistula resulted in exclusion from the study. Age, sex, otoscopic findings and audiograms were examined within each patient's file. Bone conduction thresholds (BCT) obtained on pure tone audiogram (PTA) at four different frequencies $(0.5,1,2,4 \mathrm{kHz})$, and their averages, were evaluated and compared, between the normal and diseased ear for the individuals in each group. This comparison was also made between the diseased ears

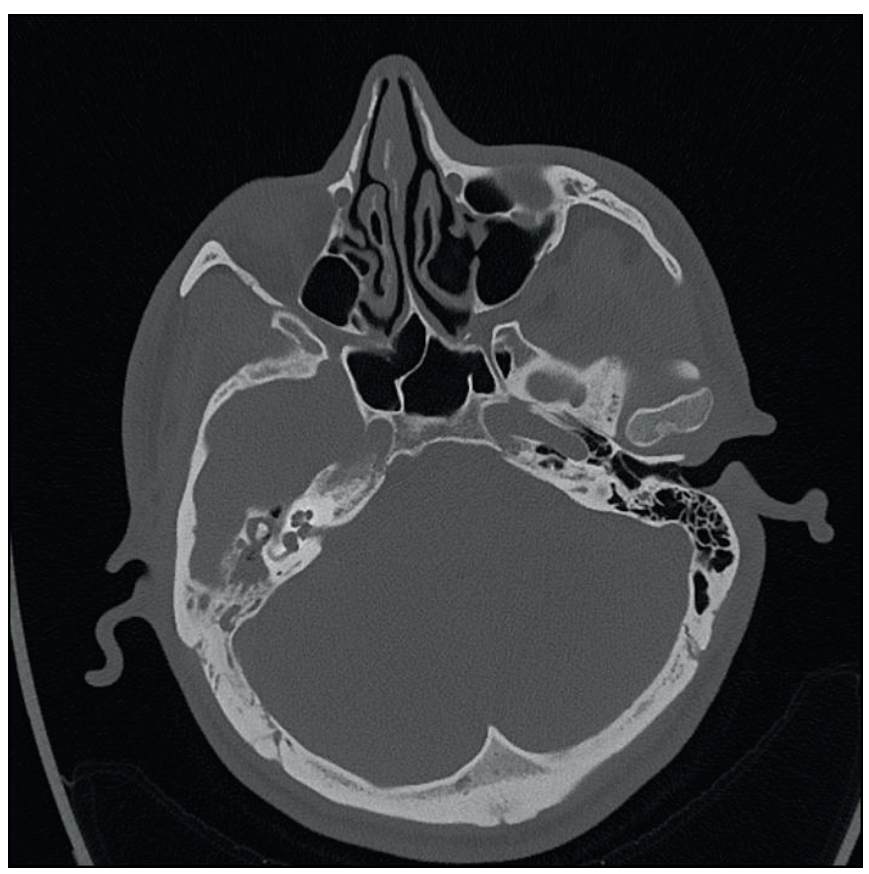

Figure 1. Axial temporal CT, unilateral COM (right).

in Groups 1 and 2. The data were analysed statistically using the Independent-Samples Kruskal-Wallis Test contained within the software (SPSS FW, SPSS Inc. Chicago, IL, USA).

\section{Results}

Group $1(\mathrm{n}=80)$ consisted of 33 men and 47 women, whereas there were 19 men and 13 women in Group $2(n=32)$. The mean age of individuals in Group 1 was $27.60 \pm 11.77$ (range:11-60) years and in Group 2 was $26.50 \pm 13.03$ (range:10-60) years.

There was a statistically significant difference between the mean BCT obtained on PTA of the normal/diseased ears in Group $1(9.78 \pm 0.98 / 17.34 \pm 1.71 \mathrm{~dB})$ and Group 2 $(9.10 \pm 0.99 / 17.58 \pm 2.59 \mathrm{~dB})$. This statistically significant difference was observed for each of the four different frequencies ( $\mathrm{p}<0.0001)$ (Table 1, Table 2). However, there was no statistically significant difference observed between the mean BCT scores obtained by PTA for the diseased ears in Groups 1 and $2(17.34 \pm 1.71$ vs $17.58 \pm 2.59 \mathrm{~dB})$. Similarly, in the comparison between the diseased ears, the differences of BCT at all four different frequencies $(0.5,1,2,4$ $\mathrm{kHz}$ ) lacked statistical significance $(\mathrm{p}>0.05)$ using the Independent-Samples Kruskal - Wallis Test (Table 3). These findings are summarized in Figure 2. 


\begin{tabular}{lccccc}
\hline \multicolumn{4}{l}{ Table 1. BCT-PTA at 4 frequencies for normal and diseased ears in Group 1. } \\
\hline Group 1 $(\mathrm{n}=\mathbf{8 0})$ & $\mathbf{0 . 5} \mathbf{~ k H z}$ & $\mathbf{1 ~ k H z}$ & $\mathbf{2 ~ k H z}$ & $\mathbf{4} \mathbf{~ k H z}$ & $\mathbf{M e a n} \mathbf{~ k H z}$ \\
\hline BCT-PTA Normal ears & $10.50 \pm 3.61 \mathrm{~dB}$ & $8.94 \pm 3.62 \mathrm{~dB}$ & $8.94 \pm 4.48 \mathrm{~dB}$ & $10.75 \pm 7.68 \mathrm{~dB}$ & $9.78 \pm 0.98 \mathrm{~dB}$ \\
BCT-PTA Diseased ears & $19.10 \pm 9.18 \mathrm{~dB}$ & $15.88 \pm 8.99 \mathrm{~dB}$ & $18.56 \pm 7.92 \mathrm{~dB}$ & $15.88 \pm 9.44 \mathrm{~dB}$ & $17.34 \pm 1.71 \mathrm{~dB}$ \\
p & $<0.0001$ & $<0.0001$ & $<0.0001$ & $<0.0001$ & $<0.0001$ \\
\hline
\end{tabular}

BCT: bone conduction thresholds, kHz: kiloHertz, PTA: Pure tone audiogram

\begin{tabular}{|c|c|c|c|c|c|}
\hline Group $2(n=32)$ & $0.5 \mathrm{kHz}$ & $1 \mathrm{kHz}$ & $2 \mathrm{kHz}$ & $4 \mathrm{kHz}$ & Mean kHz \\
\hline BCT-PTA Normal ears & $8.75 \pm 4.92 \mathrm{~dB}$ & $8.13 \pm 4.71 \mathrm{~dB}$ & $9.06 \pm 4.30 \mathrm{~dB}$ & $10.47 \pm 7.11 \mathrm{~dB}$ & $9.10 \pm 0.99 \mathrm{~dB}$ \\
\hline BCT-PTA Diseased ears & $19.69 \pm 8.51 \mathrm{~dB}$ & $14.38 \pm 6.32 \mathrm{~dB}$ & $19.69 \pm 9.58 \mathrm{~dB}$ & $16.56 \pm 8.18 \mathrm{~dB}$ & $17.58 \pm 2.59 \mathrm{~dB}$ \\
\hline $\mathrm{p}$ & $<0.0001$ & $<0.0001$ & $<0.0001$ & $<0.0001$ & $<0.0001$ \\
\hline
\end{tabular}

BCT: bone conduction thresholds, kHz: kiloHertz, PTA: Pure tone audiogram

\begin{tabular}{|c|c|c|c|c|c|}
\hline $\begin{array}{c}\text { Diseased Ears in } \\
\text { Group } 1 \text { and Group } 2\end{array}$ & $0.5 \mathrm{kHz}$ & $1 \mathrm{kHz}$ & $2 \mathrm{kHz}$ & $4 \mathrm{kHz}$ & Mean kHz \\
\hline $\begin{array}{l}\text { BCT-diseased ears Group } 1 \\
\qquad(n=80)\end{array}$ & $19.10 \pm 9.18 \mathrm{~dB}$ & $15.87 \pm 8.99 \mathrm{~dB}$ & $18.56 \pm 7.92 \mathrm{~dB}$ & $15.88 \pm 9.44 \mathrm{~dB}$ & $17.34 \pm 1.71 \mathrm{~dB}$ \\
\hline $\begin{array}{l}\text { BCT-diseased ears Group } 2 \\
\qquad(n=32)\end{array}$ & $19.69 \pm 8.51 \mathrm{~dB}$ & $14.38 \pm 6.32 \mathrm{~dB}$ & $19.69 \pm 9.58 \mathrm{~dB}$ & $16.56 \pm 8.18 \mathrm{~dB}$ & $17.58 \pm 2.59 \mathrm{~dB}$ \\
\hline$p$ & $>0.05$ & $>0.05$ & $>0.05$ & $>0.05$ & $>0.05$ \\
\hline
\end{tabular}

BCT: bone conduction thresholds, kHz: kiloHertz, PTA: Pure tone audiogram

\section{Discussion}

Chronic otitis media may cause a clinically significant SNHL without eroding the otic capsule. ${ }^{[10,11]}$ Although the explanation for this phenomenon remains controversial, it has been suggested that inflammatory mediators, lytic enzymes and toxins may penetrate the inner ear via the round-window membrane and cause destruction of the hair cells, especially in the basal turn of the cochlea where the higher frequencies are represented. ${ }^{[5,7,9,12,13]}$ The permeability of the round window membrane increases due to chronic inflammation. ${ }^{[11,14]}$ This patho- genic mechanism may explain the early appearance of an interaural difference in BCT obtained on PTA at higher frequencies $(4 \mathrm{kHz})$ in patients with unilateral COM. ${ }^{[9,13]}$ Cureoglu et al ${ }^{[7]}$ found a statistically significant loss of both inner and outer hair cells in the basal turn of the cochlea in diseased temporal bones. They also demonstrated the destruction of the stria vascularis and spiral ligament.

Blunt or penetrating otologic trauma, an occult perilymphatic fistula, drilling and ossicular manipulation from previous otologic surgery may also produce negative effects on cochlear function. ${ }^{[2,8,10]}$ In this study, in order to 


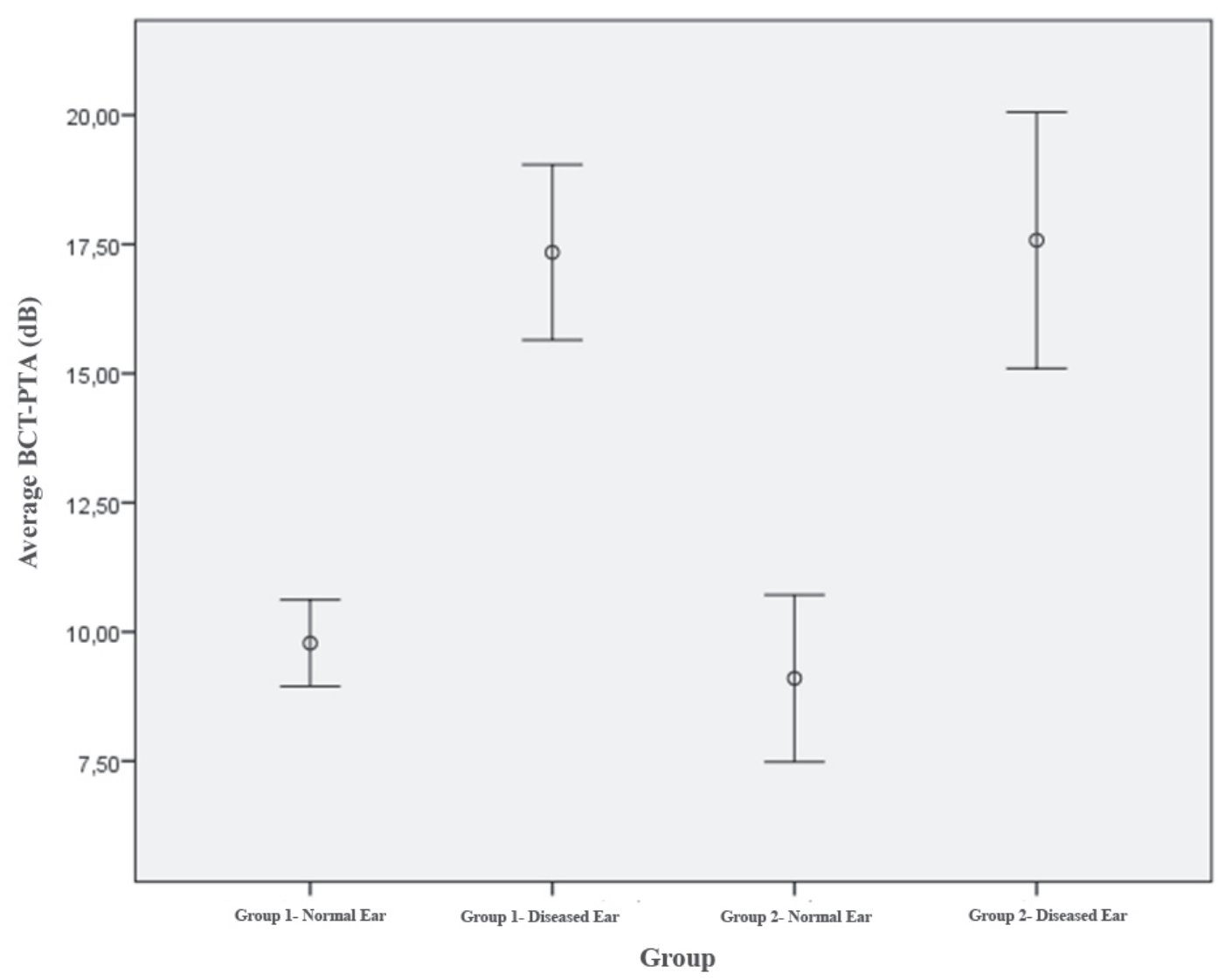

Figure 2. Mean BCT obtained on PTA for normal and diseased ears in Group 1 and Group 2.

focus on the effects of COM alone, individuals with potential SNHL from other causes, as mentioned above, were excluded.

The duration of disease and the existence of accompanying cholesteatoma may be risk factors for the development of SNHL in adult patients with COM. Cochlear damage and SNHL may be more apparent when cholesteatoma co-exists, because of the destructive nature of cholesteatoma. ${ }^{[8,9]}$ Walby et al ${ }^{[12]}$ reviewed case notes relating to the temporal bone of 87 patients with unilateral COM, to ascertain the effects of the disease on the cochlea. They report a decrease in BCT obtained on PTA in diseased ears. However, they failed to find any evidence for inflammatory penetration through the round-window membrane. There was no difference in BCT obtained on PTA between cases of COM with cholesteatoma and those without, in their study. Paparella et al ${ }^{\left[{ }^{[6]}\right.}$ noted an elevation in BCT obtained on PTA at 1, 2 and $4 \mathrm{kHz}$ in $279 \mathrm{COM}$ cases. According to the authors of that study, penetration by inflammatory cells and mediators via the round-window membrane of the inner ear was the principal cause of cochlear dysfunction. The presence or absence of cholesteatoma did not feature among the variables considered by the study.

Levine et al ${ }^{[8]}$ reported that SNHL was greater in the diseased ear at all frequencies than in the contralateral ear in patients with unilateral COM. The difference in mean BCT obtained on PTA in 161 cases was reported to be 9.1 $\mathrm{dB}$ (range:5.6-12.8 dB). In that study, the frequencies where the greatest effect was seen were 2 and $3 \mathrm{kHz}$. The authors also investigated the potential effects of varying middle ear disease severity and patient age on SNHL. They reported that $64 \%$ of the 161 cases enrolled in the study consisted of COM with cholesteatoma, and the presence of choleste- 
atoma had additional adverse effects on cochlear function. However, this observed difference was only statistically significant at a frequency of $0.5 \mathrm{kHz}$. It was also reported that there was a small, but statistically significant, effect of age on increasing the BCT.

Papp et al ${ }^{[13]}$ notes a strong correlation between patient age and BCT in COM cases. SNHL has been reported as more pronounced at higher frequencies. According to the authors, due to the proximity of the round window to the basal turn of the cochlea, inflammatory mediators, lytic enzymes and toxins which arise from the middle ear may be present at higher concentrations. Thus SNHL will occur at high frequencies first.

Azevedo et al ${ }^{[15]}$ retrospectively reviewed case notes from 115 patients with COM. Neither the presence of cholesteatoma nor the duration of disease were found to affect SNHL, however, the patient's age was identified as a significant factor. Kolo et al ${ }^{[16]}$ observed a significant degree of SNHL in diseased ears compared to normal ears. However, they did not identify any correlation between the patient's age or duration of disease and the degree of SNHL. Amali et al ${ }^{[4]}$ report that a statistically significant difference in mean BCT was obtained on PTA between normal and diseased ears at all frequencies. The authors found that there was a significant correlation between age and SNHL, but that the duration of the disease did not have a significant effect on SNHL. Furthermore, they report that there is no relationship between the presence of cholesteatoma and the degree of SNHL.

In the present study, a statistically significant difference in BCT obtained on PTA between the normal and the diseased ears in both groups was found for all 4 frequencies tested $(\mathrm{p}<0.0001)$. However, in the comparison between the diseased ears, the differences of BCT at all four different frequencies $(0.5,1,2,4 \mathrm{kHz})$ lacked statistical significance $(p>0.05)$. Ninety percent of our patient sample was under 50 years of age, and the duration of COM was similar, therefore the data were unsuitable for investigating any potential effect of patients' age and duration of disease on BCT.

Our study suffers from certain limitations. The possible effects of age and disease duration on BCT were not evaluated. Moreover, the number of patients included in the study was relatively small.

\section{Conclusion}

According to the results obtained in this study, it can be stated that COM may cause SNHL. However, the presence of cholesteatoma does not result in a statistically significant additional negative impact on cochlear function. Surgical management of COM should be routinely recommended to patients in order to circumvent serious complications of COM, such as SNHL.

\section{Acknowledgements: None}

Ethics Committee Approval: All procedures involving human participants were in accordance with the ethical standards of the Necmettin Erbakan University Meram Medical Faculty (Ethics Committee Decision - 017/777)

Informed Consent: Informed consent was not required due to the retrospective study design.

Author Contributions: Designing the study - C.H.U.; Collecting the data - C.H.U., D.A., A.Y., F.A.; Analys-ing the data - D.A., A.Y., F.A.; Writing the manuscript - C.H.U., D.A.; Confirming the accuracy of the data and the analyses - C.H.U., D.A., A.Y., F.A.

Conflict of Interest: The authors have no conflicts of in-terest to declare.

Financial Disclosure: The authors declared that this study has received no financial support. 


\section{References}

1. Silveira Netto LF, da Costa SS, Sleifer P, Braga ME. The impact of chronic suppurative otitis media on children's and teenagers' hearing. Int J Pediatr Otorhinolaryngol 2009;73:1751-6.

2. Yehudai N, Most T, Luntz M. Risk factors for sensorineural hearing loss in pediatric chronic otitis. Int J Pediatr Otorhinolaryngol 2015;79:26-30.

3. Aarhus L, Homøe P, Engdahl B. Otitis Media in Childhood and Disease in Adulthood: A 40-Year Follow-Up Study. Ear Hear 2019 Apr 16. doi: 10.1097/AUD. 000000000000729.

4. Amali A, Hosseinzadeh N, Samadi S, Nasiri S, Zebardast J. Sensorineural hearing loss in patients with chronic suppurative otitis media: Is there a significant correlation? Electron Physician 2017;9:3823-7.

5. Paparella MM, Goycoolea MV, Schachern PA, Sajjadi H. Current clinical and pathological features of round window diseases. Laryngoscope 1987;97:1151-60.

6. Paparella MM, Brady DR, Hoel R. Sensori-neural hearing loss in chronic otitis media and mastoiditis. Trans Am Acad Ophthalmol Otolaryngol 1970;74:108-15.

7. Cureoglu S, Schachern PA, Paparella M, Lindgren R. Cochlear changes in chronic otitis media. Laryngoscope 2004;114:622-6.

8. Levine BA, Shelton C, Berliner KI, Sheehy JL. Sensorineural loss in chronic otitis media. Is it clinically significant? Arch Otolaryngol Head Neck Surg 1989;115:814-6.

9. Eisenman DJ, Parisier SC. Is chronic otitis media with cholesteatoma associated with neurosensory hearing loss? Am J Otol 1998;19:20-5.

10. Tos M. Sensorineural hearing loss in acute and chronic middle ear diseases. Acta Otolaryngol Suppl 1989;457:87-93.

11. da Costa SS, Rosito LPS, Dornelles C. Sensorineural hearing loss in patients with chronic otitis media. Eur Arch Otorhinolaryngol 2009:266;221-4.

12. Walby AP, Barrera A, Schuknecht HF. Cochlear pathology in chronic suppurative otitis media. Ann Otol Rhinol Laryngol Suppl 1983;103:119.

13. Papp Z, Rezes S, Jo'kay I, Sziklai I. Sensorineural hearing loss in chronic otitis media. Otol Neurotol 2003;24:141-4.

14. MacAndie C, O'reilly B.F. Sensorineural hearing loss in chronic otitis media. Clin Otolaryngol 1999;24:220-2.

15. de Azevedo AF, Pinto DC, de Souza NJ, Greco DB, Gonçalves DU. Sensorineural hearing loss in chronic suppurative otitis media with and without cholesteatoma. Braz J Otorhinolaryngol 2007;73:671-4.

16. Kolo ES, Salisu AD, Yaro AM, Nwaorgu OG. Sensorineural hearing loss in patients with chronic suppurative otitis media. Indian J Otolaryngol Head Neck Surg 2012;64:59-62. 